\title{
MEASUREMENT OF THE TREAD MOVEMENT OF PNEU. MATIC TIRES AND A DISCUSSION OF THE PROBABLE RELATION TO TREAD WEAR
}

\author{
By W. L. Holt and C. M. Cook
}

\begin{abstract}
From an observation of the ways in which pneumatic-tire treads wear in service, it appears that the movement of the tread when in contact with the road is probably an important cause of wear. In this paper two methods are described for recording tread movements as a tire rolls along under load. One way, which is referred to as the wax-plate method, is particularly adapted to this work because the tread movements can be recorded quickly and easily with very simple equipment. The results of several tests by this method are shown, and some of the factors in tire construction and operation which affect tread movements are determined. Methods for the reduction of this tread movement are suggested which should have a direct bearing on the reduction of tread wear.
\end{abstract}

\section{CONTENTS}

I. Introduction

III. Results with different tires

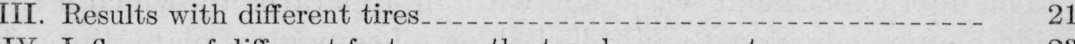

IV. Influence of different factors on the tread movements._._.

V. Discussion of results____._. 25

VI. Conclusions

\section{INTRODUCTION}

In this investigation a study of the manner in which pneumatic-tire treads wear has been made by observing the movement of the tread when in contact with a flat surface. The matter of tread wear has come very much to the front since the general adoption of the balloon tire, which, because of its greater deflection and larger tread contact area, presents a more serious problem in this respect than the highpressure tire. The thought which is being given to this problem is illustrated by the many references to "flat treads," "round treads," "center hinges," "riding strips," etc. Under normal conditions a tire should be expected to wear uniformly and symmetrically around its periphery, but it is a matter of general observation that 
treads do not always wear in this way. Some of the common ways of wearing nonuniformly are "cupping" of treads on each side of the center line, wearing of the edges of the buttons, and nonsymmetrical wear with respect to the center line of the tire. Accordingly, it was felt that a study of the way in which treads are abraded should. yield valuable information concerning the causes of tread wear, and thus indicate the most economical use of rubber in the tread.

\section{METHODS TRIED}

Tread movements were observed and recorded by several different methods.

One method made use of a revolving slotted disk driven by an electric motor, the speed of which could be controlled. A tire was mounted on an ordinary type of test machine in which the tire revolves against a metal drum. By mounting the revolving disk close to the contact surface of the tire and the drum and adjusting the speeds properiy the tread movement just before coming in contact with the drum could be observed. However, it was not possible to obtain data as to what occurred after the tire came in contact with the drum.

Another method tried was to coat the tire tread with a white enamel sufficiently flexible so that it would not chip. The tire was run for a short time against an abrasive surface with the thought that movements of the tread would show up by corresponding scratches on the enamel. The results of a trial, however, did not look promising and the plan was abandoned.

The next experiment was made with an apparatus by which a tire could be pressed against and rolled along a heavy glass plate, through which the tread movement could be observed. This apparatus is shown in Figure 1. The tire is mounted on the shaft of the motor simply for convenience, as the motor itself is not used. A loading device permits the tire to be pressed against the plate glass, which is secured to a small carriage. The carriage can be moved up and down by turning the handle which operates through a rack and pinion. Thus the action of a tire in rolling along a flat surface is simulated. The glass plate is ruled and etched in 1-inch squares as reference lines. It would probably be desirable to subdivide these into one-tenth inch over at least the central portion of the glass. By noting successive positions of any particular point on the tire with respect to a reference point on the glass the movement of the tread rubber can be studied. In order to show up the area in contact plainly it is advisable to coat the glass with a liquid, such as a soap solution or glycerin. If desired, photographs can be taken through this glass as the tire rolls. (See fig. 2.) Visual observations, however, may furnish 
B. S. Journal of Research, RP2

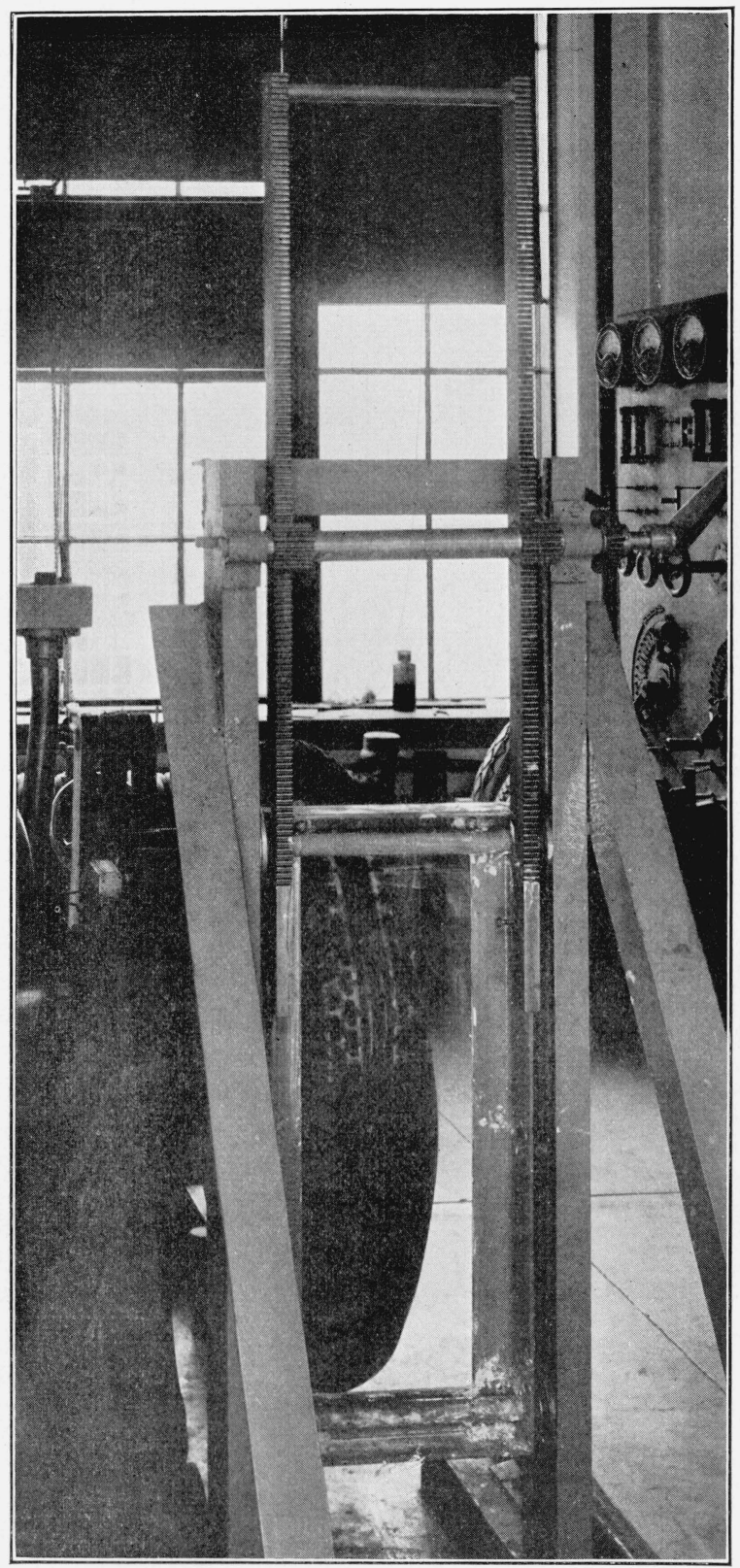

FIG. 1.-A pparatus used for observing tread movements through a glass plate 
B. S. Journal of Research, RP2

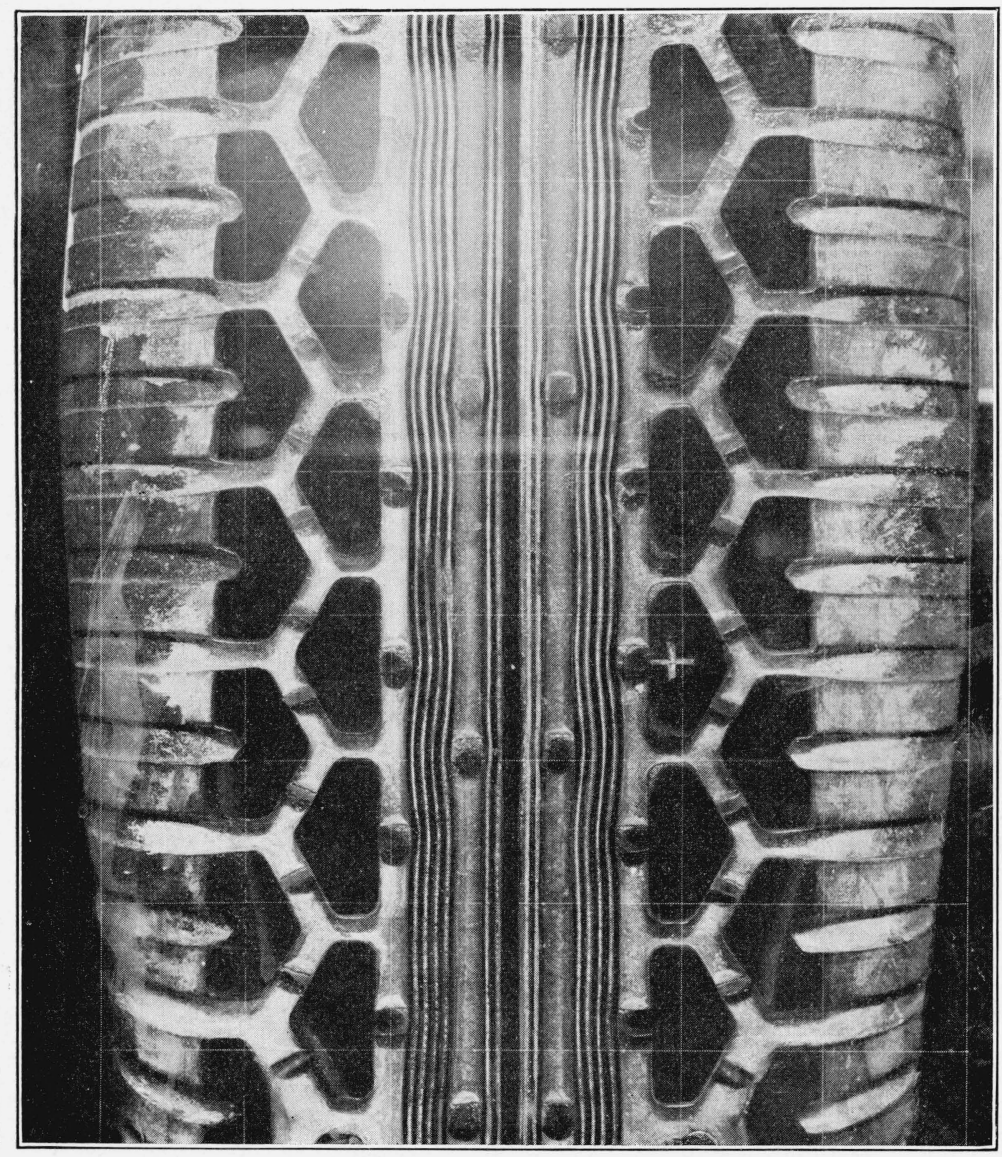

FIG. 2.-Photograph taken through the plate glass showing the contact area of a tire under load

(The depressions in the tread were painted white to show contrast) 
all the information that is desired. In obtaining data which could be plotted as graphs, a telescope was mounted about 6 feet away, and observations made through it. This aided considerably in following the movement of particular points in the tread.

Observations of several tires showed that different parts of the tread had characteristic movements. For instance, the central part of a tire usually showed straight-line movements opposite to the direction of travel of the tire, while other parts had a curved movement with a component of motion in the same direction as that in which the tire traveled.

Plotting tread movements in this way, however, is a rather tedious process; therefore, a quicker method was tried which proved to be very satisfactory for recording the complete movement of any point on the tread. This method consists of recording the movement on waxed plates and is carried out as follows: Aluminum sheets about 8 by 10 inches and 0.010 inch thick are given a thin coating of melted paraffin wax on one side. Several different waxes were tried out, but paraffin was found to give the best results at ordinary room temperature. Harder waxes were not satisfactory. After the wax has set, the surface is sprinkled very sparingly with grains of No. 60 carborundum. As a tire under load flexes when rolled over one of these plates, the carborundum grains (or at least a portion of them) stick to the tire and trace their path on the waxed plate. It is well to roughen the surface of the tread slightly (as with sand or emery paper) to remove any glaze, and give a better chance for the grains to stick.

Figure 3 shows photographs taken of a typical wax-plate record. The straight lines in the central part of the treads and the more or less curved lines in other parts were traced by the individual grains of carborundum.

Records were made from several tires by using the apparatus shown in Figure 1, and interposing the waxed plates between the tire and the glass. However, it was found that practically the same records could be obtained by using an ordinary drum tire testing machine and allowing the waxed plates to pass between the tire and the drum. This offered several advantages because in addition to being more convenient it permitted records to be made under normal conditions of speed and tractive effort.

\section{RESULTS WITH DIFFERENT TIRES}

Different tires were mounted on a drum test machine ${ }^{1}$ and records made of the tread movements under air pressures and axle loads corresponding to normal operating conditions. The tire was turned by hand at a speed corresponding to about 2 miles per hour as the waxed plate passed between the tire and the drum. From these

\footnotetext{
1 The dynamometers which are described in B. S. Tech. Paper No. 213, Powe: Losses in Tires, were used.
} 
records, the line drawings shown in Figure 4 were made. The are above each section shows the profile of the raised portion of the tread. These are not intended to be absolute reproductions of tread designs or tread movement, but rather to show what may be expected from different tires and the approximate movements of points in the tread.
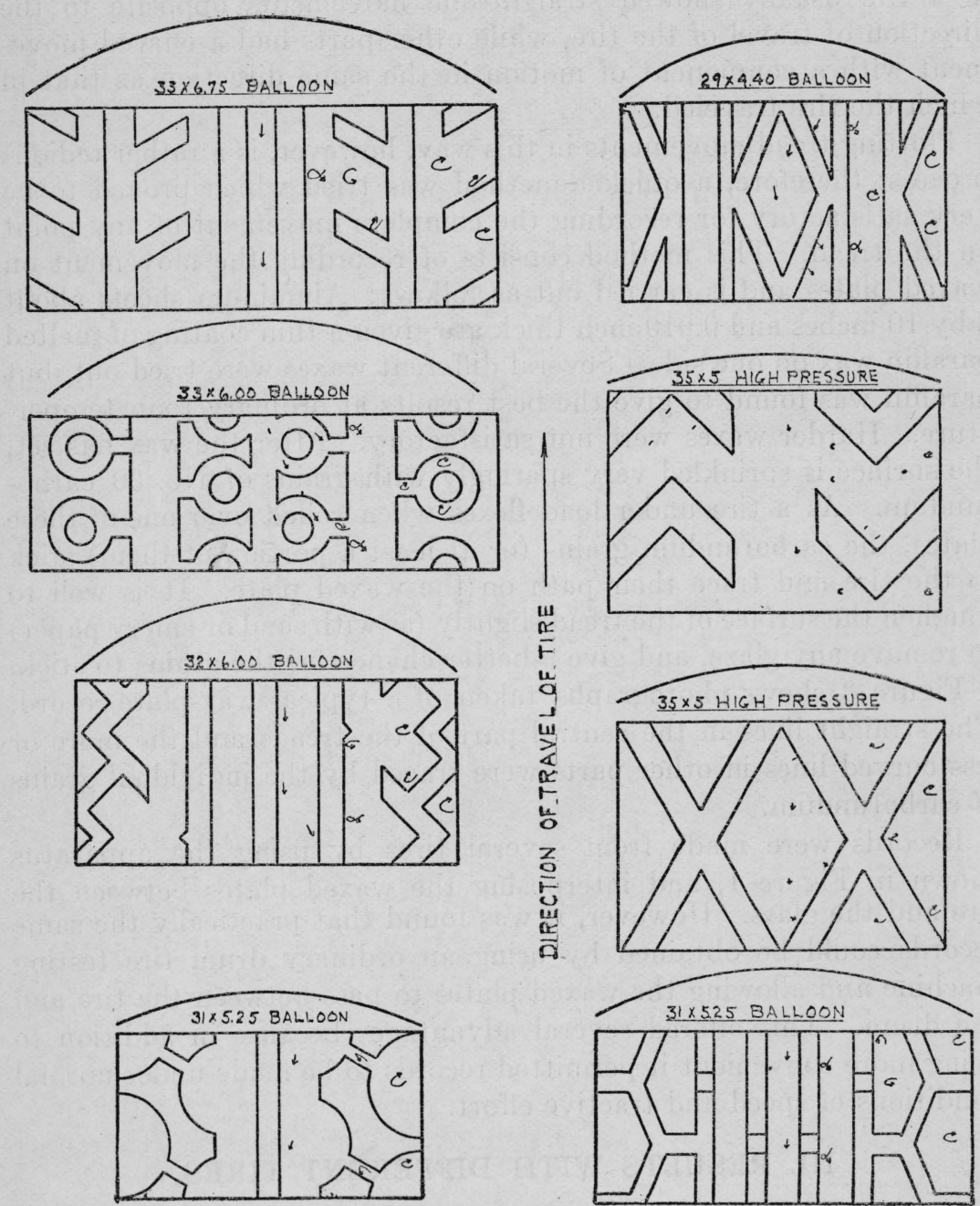

FIG. 4.-Drawings taken from wax records showing tread movements of different tire treads

It will be noted that the general movements in all the treads are similar. Each tread may be divided into three parts - the center, the intermediate portion, and the outer portion. In the center of the tread the movement is in an approximate straight line along the 
B. S. Journal of Research, RP2

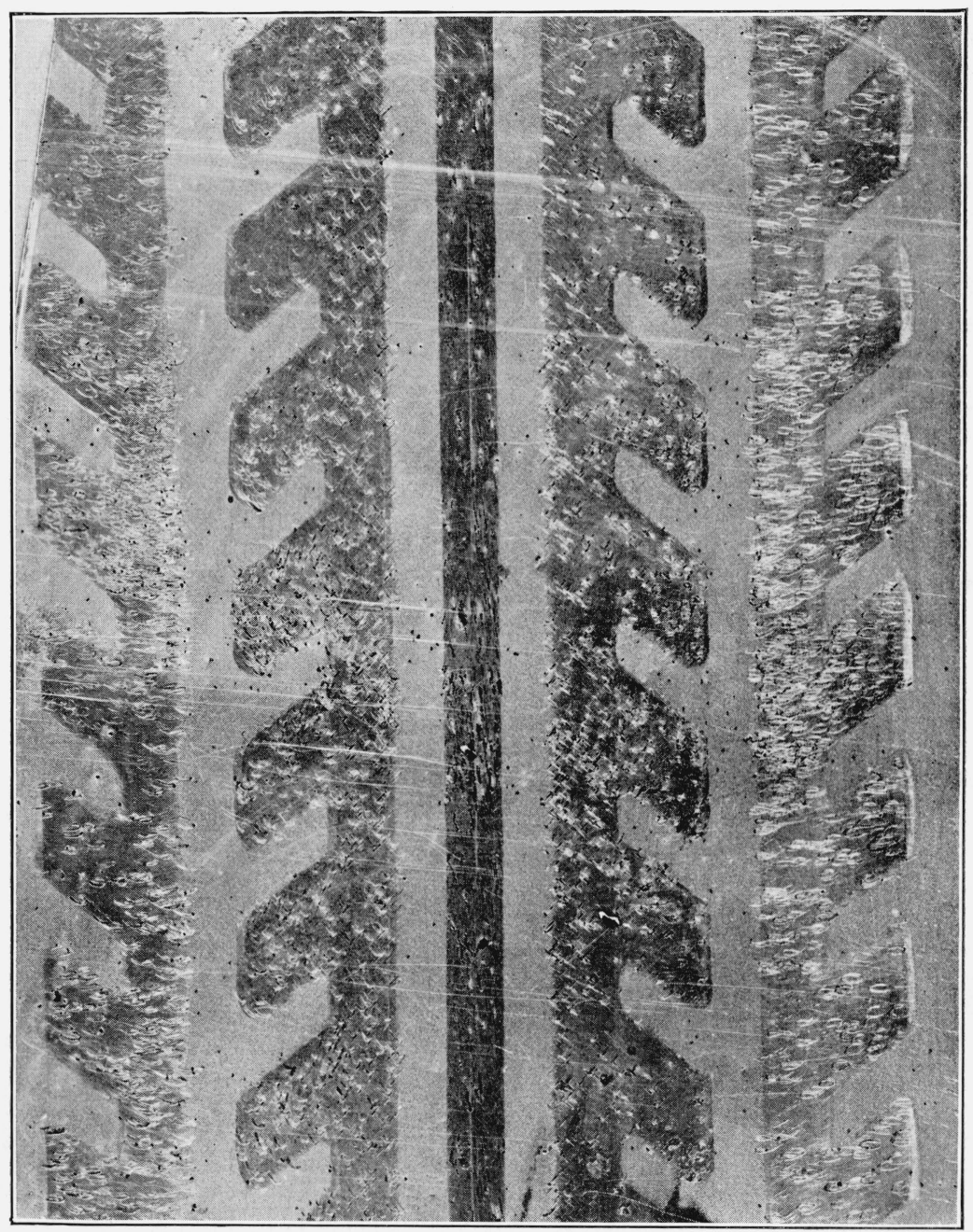

Fig. 3.-Typical wax plate showing tread movements 
circumference in a direction opposite to the direction in which the tire travels. The outer portions of the tread also have circumferential movements, but in the direction of travel. In addition, they move toward and away from the center line, thus giving the curves observed. The intermediate portions move similarly, but the resultant movement may be either in the direction of travel or against it, depending largely on the distance from the center line. A V-shaped mark on a wax plate is a common record from this part of the tread.

\section{INFLUENCE OF DIFFERENT FACTORS ON THE TREAD MOVEMENTS}

The question is apt to arise as to whether the results made by simple rolling tests at very low speeds are comparable with those obtained at higher speeds. As noted previously, the method of recording on waxed plates permits of records being made at a considerable tire speed. Records were made on tires running at speeds as high as 30 miles per hour. The waxed plate was simply dropped between the tire and the drum and allowed to fall on the floor after passing through. The treatment was a little severe, but in most cases the wax record was not damaged. The results seemed to be entirely comparable with those obtained from the same tire at very low speeds.

Some records were also made using a tire running at a speed of 22 miles per hour while transmitting 0,4 , and 8 horsepower, respectively, corresponding to tractive efforts of approximately 0,70 , and 140 pounds. The results are illustrated in Figure 5.

The scratches in the center of the tread are increased in length as the tractive effort is increased, which is in accordance with what is to be expected. The circumferential movement of points in the intermediate portions of the tread are increased while circumferential movements of the outer portions (which are normally in the direction of travel) are decreased. The large movement, however, toward and away from the center does not appear to change appreciably with a change in tractive effort. A large tractive effort, such as might be produced by a sudden application of the brakes of an automobile or very rapid acceleration, would be outside the scope of this study.

The air pressure and the axle load under which a tire is operated affect the tread movement considerably. The greatest tread movements occur under low air pressures or high-axle loads. The movement, however, appears as simply an exaggeration of that which occurs under normal operating conditions and increases with increased. flexing. Figure 4 shows both high pressure and balloon tires. The movement is more pronounced in the balloon tire, which is in accord- 
ance with the common observation that the treads of balloon tires are inclined to wear faster and less uniformly than the treads of high-pressure tires.

The common practice of cambering and toeing in of the front wheels of an automobile doubtless influences the tread wear. It

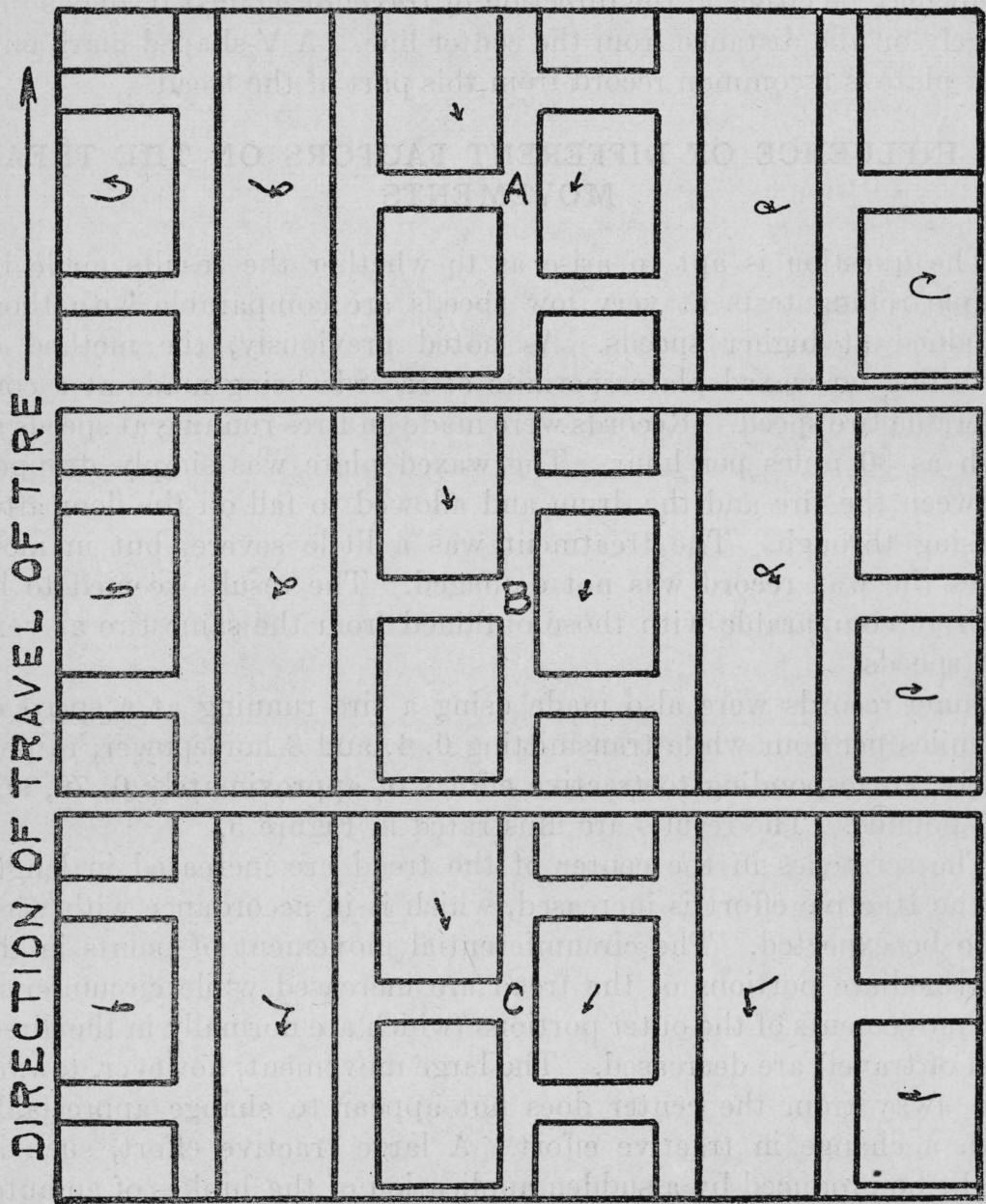

FIG. 5.-Drawings taken from wax records showing the effect of tractive effort on tread movements

A.- - No tractive effort.

B. -70 pounds tractive effort.

C. -140 pounds tractive effort.

should not be difficult to determine the effect of these factors on the tread movements either in the laboratory or on the road by the use of waxed plates.

The results of these tests indicate that the movement of a tread may be affected by the direction of rotation, particularly in the case 
of a nonsymmetrical design. It was noted that with some tires the tread movement is excessive at certain points, particularly at the ends or edges of nonskid buttons. From an observation of the way in which a button comes in contact with and leaves the ground, it would seem desirable to design the buttons so that the edges would be approximately parallel to the periphery of the area of tire contact. This would tend to make the total button come into contact quickly after once touching.

Aside from indicating some of the causes of tread wear, this study points to the desirability of occasionally changing the direction of rotation of tires in service.

There has been a tendency recently to make tire treads in which the design is comparatively open in the center and which have more or less continuous "riding strips" some distance out from the center. An attempt was made to obtain data on the change in tread movements due to increasing or decreasing what might be called the rigidity of the center of the tire. The results are illustrated in Figure 6. Four different degrees of rigidity are shown which were produced either by cutting additional depressions in the tread or by filling some of the depressions of the original design with strips of rubber. This naturally did not give as rigid a condition as would result from a homogeneous mass, but should approximate this condition. A shows the most flexible tread condition while $D$ shows the most rigid condition. The movements of the intermediate and outer portions of the treads shown in $A, B$, and $C$ seem about the same. The center movements are somewhat erratic. In $A$ the center movement apparently depends on whether or not a button as a whole is distorted without slipping. In $B$ there seems to be an increased tendency to crowd toward the center depression. In $C$, on account of the increased. rigidity in a circumferential direction, there seems to be a tendency to crowd away from the center. In $D$, the movement of the whole central portions is less than in the other cases, but the outside movement is increased.

In Technologic Paper No. 240 (Dynamometer Tests of Automobile Tires) it was shown that the creep of a tire is dependent on the carcass design as well as on the tread design. This indicates that the tread movement is affected by the carcass as well as by the tread configurations.

\section{DISCUSSION OF RESULTS}

The results obtained by recording the tread movements indicate that at least a considerable part of tread wear may be caused by the slipping of portions of the tread over the road surface in changing from the normal to the deflected condition and vice versa. This might be termed a scuffing action. It is recognized that a tire seldom rolls 


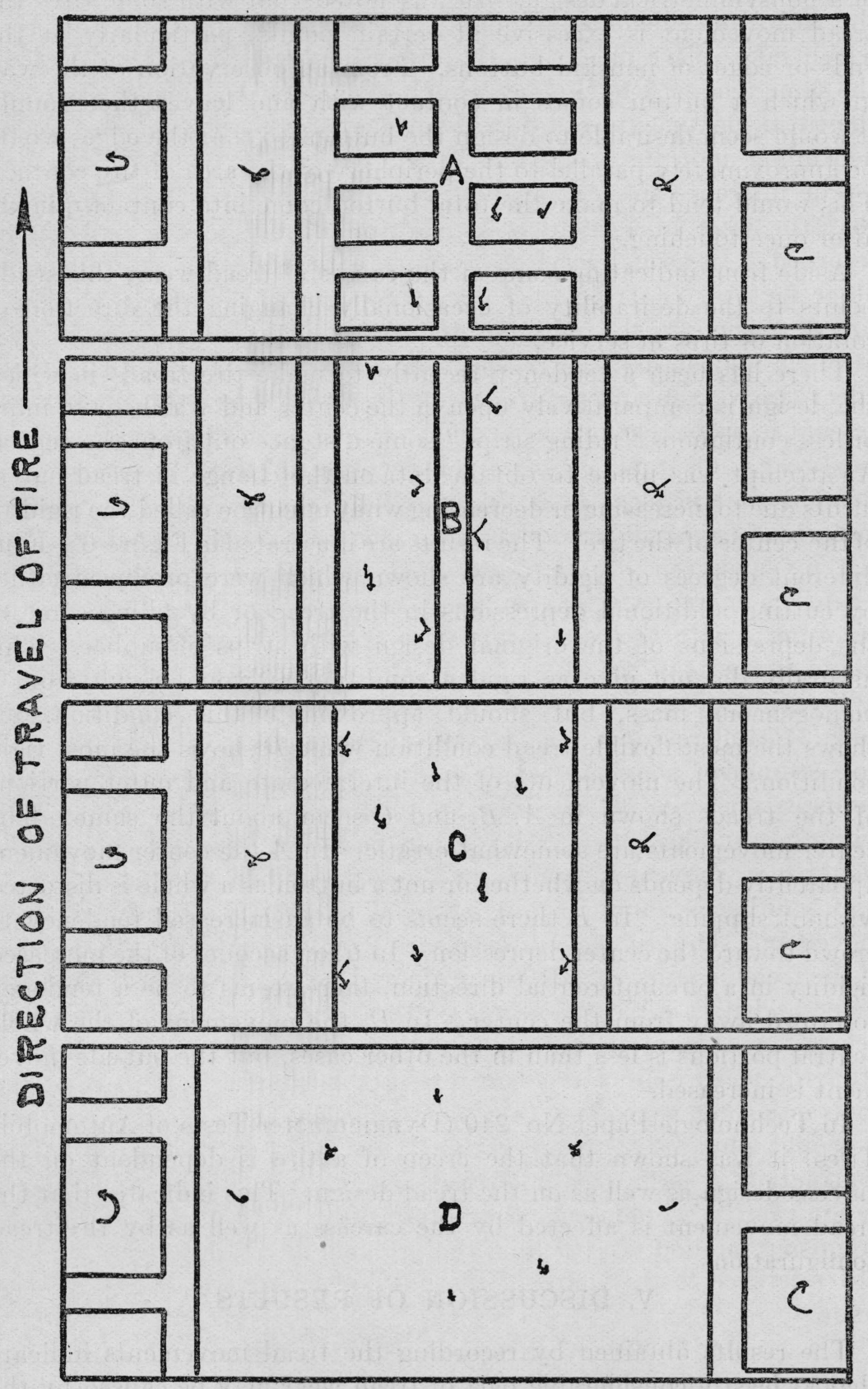

FIG. 6.-Drawings taken from wax records showing the effect of changing the rigidity of the center portion of a tire tread on the tread movements 
over a surface as smooth as a piece of glass or a waxed plate and that the tread movement on a rough surface may be different from that which takes place on a smooth surface. Nevertheless there will be the same tendency to slip on a rough surface as on a smooth one, and the tread movement on different surfaces will simply vary in degree, depending upon the coefficients of friction.

The inference should not be drawn from the preceding discussion that the wear of a tread is attributed entirely to the movements recorded. In actual service, of course, tread wear is dependent on other factors. For instance, high tractive efforts which cause the tire as a whole to slip, slipping due to braking, the bouncing of tires in which they actually leave the ground, and side skidding. All these affect tire wear in addition to the suggested scuffing action. In some cases it is probable that these items are the predominating factors in tire wear, but from a general observation of tires in service it is thought that in most cases scuffing is the important, or at least an important factor in producing wear. A few examples will perhaps aid in substantiating this belief: (1) The tendency of treads to "cup" is more prevalent in front than in rear tires, which is in accordance with the conclusions that tractive effort tends to reduce the movements of the outer tread and to increase center movement; (2) wearing of the edges of the buttons is obviously a scuffing action; and (3) a microscopic examination of the surface of used tires does not ordinarily show abrasion in any particular direction except, perhaps, on some of the edges of buttons. This is in accordance with the scuffing theory. Wear due to slip of the tire as a whole would show movement mainly in a circumferential direction.

If scuffing is an important cause of tire wear, a study of tread movements should have a direct bearing on the subject. The portion of the tread in contact with the ground is contracted from the normal shape and it is noted that some of this contraction takes place just before the tread touches the ground. The remainder takes place after the tread is in contact, and results in slipping. If more of the contraction can be made to take place when the tread is out of contact, it would obviously reduce slipping and thus reduce wear. This contraction is influenced by tread design, tread shape, and carcass design, and it would seem that a study of this contraction offers a promising method of attacking the problem of tread wear.

A reference to the shape of the nonskid buttons has already been made.

It was noted in several tires that there is a tendency for the tread to slip nonsymmetrically even though the tread design is symmetrical. At first this was attributed to the tire or rim being out of alignment. A closer examination and adjustment of the apparatus, however, 
indicates that this is the result of some nonuniformity in the tire itself.

\section{CONCLUSIONS}

Two methods have been described which have been successfully tried for observing the movement of the treads of pneumatic tires under running conditions. By the second method quite complete and reliable data can be obtained with very simple equipment. This study indicates that tread movement is probably a fundamental cause of tire-tread wear and points out how certain conditions of operation affect this movement. If desired, tread movements can be observed by one of the methods with a tire running at a considerable speed and under a tractive effort. Tread movements at low speeds and no tractive efforts, however, seem to yield most of the information desired. These results show that a study of the tread movements may yield valuable information concerning the uniformity of construction of a tire. In developing laboratory abrasion tests to simulate road wear this work shows that it is desirable to use a nonlinear abrasive movement, since most of the tread surface is abraded by a more or less curved motion.

Washington, March 24, 1928. 